\title{
Initial comparative analysis of pulmonary involvement on HRCT between vaccinated and non-vaccinated subjects of COVID-19
}

\author{
Ashish Verma ${ }^{1} \cdot$ Ishan Kumar ${ }^{1}$ (1) $\cdot$ Pramod Kumar Singh $^{1} \cdot$ Mohammad Sharoon Ansari $^{1} \cdot$ Harsh Anand Singh $^{1}$. \\ Shashank Sonkar ${ }^{1} \cdot$ Adity Prakash $^{1} \cdot$ Ritu Ojha $^{1} \cdot$ Ram Chandra Shukla $^{1}$
}

Received: 7 July 2021 / Revised: 19 November 2021 / Accepted: 21 November 2021 / Published online: 12 January 2022

(c) The Author(s), under exclusive licence to European Society of Radiology 2022

\begin{abstract}
Objectives To compare the high-resolution computed tomography (HRCT)-derived severity score in COVID-19 patients between those who had earlier received the vaccine against the SARS-CoV-2 and those who did not.

Methods A retrospective cross-sectional analysis of HRCT of the chest was done in correlation with the vaccination status of clinically diagnosed COVID-19 patients. The variable under evaluation was the CT severity score, whereby differential analysis of the variability on this parameter between incompletely (single dose) vaccinated, completely (both doses) vaccinated, and non-vaccinated individuals was the outcome.

Results The analysis included 826 patients of which 581 did not receive any vaccination whereas 196 patients received incomplete (single dose) vaccination and 49 received complete vaccination. Mean CT severity score was lower in completely vaccinated patients $(3.5 \pm 6.3)$ vis-à-vis incompletely vaccinated $(10.1 \pm 10.5)$ and non-vaccinated $(10.1 \pm 11.4)$ individuals. The mean CT score was significantly lower in completely vaccinated patients of lower ages ( $\leq 60$ years) compared to patients above that age. The incidence of severe disease (CT score $\geq 20)$ was significantly higher in the incompletely vaccinated and non-vaccinated patients compared to that in the completely vaccinated group.

Conclusions CT severity scores in individuals receiving both doses of SARS-CoV-2 vaccination were less severe in comparison to those receiving a single dose of vaccine or no vaccine at all.

Key Points

- Patients who received complete two doses of vaccination had significantly low mean CT scores compared to the partially vaccinated patients and non-vaccinated patients.

- The mean CT scores were significantly lower in completely vaccinated patients of lower ages $(<60$ years $)$ while patients $>60$ years did not show significantly different CT scores between the vaccinated and non-vaccinated groups.

- Consolidations and ground-glass opacities were significantly lower in the group receiving complete vaccination as compared to the unvaccinated and incompletely vaccinated patients.
\end{abstract}

Keywords Tomography, X-ray computed $\cdot$ COVID-19 $\cdot$ SARS-CoV-2 $\cdot$ Vaccination

\section{Abbreviations}

COVID-19 Coronavirus disease 19

HRCT High-resolution computed tomography

Ashish Verma and Ishan Kumar are first co-authors as their contribution is equal.

Ishan Kumar

ishanjd@gmail.com

1 Department of Radiodiagnosis and Imaging, Institute of Medical Sciences, Banaras Hindu University, Varanasi 221005, India
RT-PCR Reverse transcriptase-polymerase chain reaction

SARS-CoV-2 Severe acute respiratory syndrome coronavirus 2

\section{Introduction}

The role of HRCT scan in making a diagnosis of COVID-19 has been emphasized as being a problem-solving modality in patients with complications, diagnostic dilemmas, and poor response to therapy [1-4]. Further, a more viable role may be in assessing the volume of lung involved in a confirmed 
patient and correlating the disease severity with a CT scan severity score [5-7]. Thirdly, with the upcoming of newer treatment modalities, drugs, and the vaccine, the same may be used to demonstrate the efficacy of a specific treatment on the actual morphological response in the pulmonary parenchyma. The fact remains that demonstration of less severe scores on CT scan following a specific intervention would offer a high level of confidence in favor of that modality. The aim of the present study was to compare the high-resolution computed tomography (HRCT)-derived severity scores in COVID-19 patients between those who had earlier received the vaccine against the SARS-CoV-2 and those who did not.

\section{Patients and methods}

\section{Patients}

This was a retrospective observational study done from 12 to 30 April 2021, using imaging data generated during routine clinical management with the informed consent from the patients, and approval from Institutional Ethical Committee has been taken. Non-disclosure of patients' privacy has been ensured during the scripting. Patients with the diagnosis of COVID-19 based on clinical suspicion and a positive reverse transcriptase-polymerase chain reaction (RT-PCR) who underwent an HRCT scan of the chest in our hospital were included in the study. A single-point evaluation of the scan performed between the fifth and eighth day from the onset of clinical symptoms was done, while all preceding or followup scans were excluded from the study. HRCT was done for diagnostic workup where RT-PCR results were delayed or initial RT-PCR was negative but there was high suspicion of COVID 19 infection. In RT-PCR-positive patients, CT was performed to assess CT severity and guide therapeutic management. Scans showing significant background pre-existing/ chronic pulmonary parenchymal diseases were excluded. The vaccinated patients had received either the inactivated virus vaccine BBV152 viz. Covaxin ${ }^{\circledR}$ (Bharat Biotech) or the non-replicating viral vector vaccine AZD1222 (ChAdOx1)

Table 1 CT severity score (40-point scoring system) for pulmonary involvement of COVID 19 on HRCT

\begin{tabular}{|c|c|c|c|c|c|c|c|}
\hline Right lung & Segment & Involvement & Score & Left lung & Segment & Involvement & Score \\
\hline \multirow[t]{9}{*}{ Right upper lobe } & \multirow[t]{3}{*}{ Apical } & $0 \%$ & 0 & \multirow[t]{9}{*}{ Left upper lobe } & \multirow[t]{3}{*}{ Apical } & $0 \%$ & 0 \\
\hline & & $<50 \%$ & 1 & & & $<50 \%$ & 1 \\
\hline & & $\geq 50 \%$ & 2 & & & $\geq 50 \%$ & 2 \\
\hline & \multirow[t]{3}{*}{ Anterior } & $0 \%$ & 0 & & \multirow[t]{3}{*}{ Anterior } & $0 \%$ & 0 \\
\hline & & $<50 \%$ & 1 & & & $<50 \%$ & 1 \\
\hline & & $\geq 50 \%$ & 2 & & & $\geq 50 \%$ & 2 \\
\hline & \multirow[t]{3}{*}{ Posterior } & $0 \%$ & 0 & & \multirow[t]{3}{*}{ Posterior } & $0 \%$ & 0 \\
\hline & & $<50 \%$ & 1 & & & $<50 \%$ & 1 \\
\hline & & $\geq 50 \%$ & 2 & & & $\geq 50 \%$ & 2 \\
\hline \multirow[t]{6}{*}{ Right middle lobe } & \multirow[t]{3}{*}{ Medial } & $0 \%$ & 0 & \multirow[t]{6}{*}{ Lingular segments } & \multirow[t]{3}{*}{ Superior } & $0 \%$ & 0 \\
\hline & & $<50 \%$ & 1 & & & $<50 \%$ & 1 \\
\hline & & $\geq 50 \%$ & 2 & & & $\geq 50 \%$ & 2 \\
\hline & \multirow[t]{3}{*}{ Lateral } & $0 \%$ & 0 & & \multirow[t]{3}{*}{ Inferior } & $0 \%$ & 0 \\
\hline & & $<50 \%$ & 1 & & & $<50 \%$ & 1 \\
\hline & & $\geq 50 \%$ & 2 & & & $\geq 50 \%$ & 2 \\
\hline \multirow[t]{15}{*}{ Right lower lobe } & \multirow[t]{3}{*}{ Superior } & $0 \%$ & 0 & \multirow[t]{15}{*}{ Left lower lobe } & \multirow[t]{3}{*}{ Superior } & $0 \%$ & 0 \\
\hline & & $<50 \%$ & 1 & & & $<50 \%$ & 1 \\
\hline & & $\geq 50 \%$ & 2 & & & $\geq 50 \%$ & 2 \\
\hline & \multirow[t]{3}{*}{ Anterior basal } & $0 \%$ & 0 & & \multirow[t]{3}{*}{ Anterior basal } & $0 \%$ & 0 \\
\hline & & $<50 \%$ & 1 & & & $<50 \%$ & 1 \\
\hline & & $\geq 50 \%$ & 2 & & & $\geq 50 \%$ & 2 \\
\hline & \multirow[t]{3}{*}{ Medial basal } & $0 \%$ & 0 & & \multirow[t]{3}{*}{ Medial basal } & $0 \%$ & 0 \\
\hline & & $<50 \%$ & 1 & & & $<50 \%$ & 1 \\
\hline & & $\geq 50 \%$ & 2 & & & $\geq 50 \%$ & 2 \\
\hline & \multirow[t]{3}{*}{ Lateral basal } & $0 \%$ & 0 & & \multirow[t]{3}{*}{ Lateral basal } & $0 \%$ & 0 \\
\hline & & $<50 \%$ & 1 & & & $<50 \%$ & 1 \\
\hline & & $\geq 50 \%$ & 2 & & & $\geq 50 \%$ & 2 \\
\hline & \multirow[t]{3}{*}{ Posterior basal } & $0 \%$ & 0 & & \multirow[t]{3}{*}{ Posterior basal } & $0 \%$ & 0 \\
\hline & & $<50 \%$ & 1 & & & $<50 \%$ & 1 \\
\hline & & $\geq 50 \%$ & 2 & & & $\geq 50 \%$ & 2 \\
\hline Total scores (right lung) & & & 20 & Total scores (left lung) & & & 20 \\
\hline
\end{tabular}


viz. Covishield $®$ (AstraZeneca, University of Oxford). The clinical severity of the disease was assigned at the time of CT scan and patients with $\mathrm{SpO}_{2}<94 \%$ on room air, respiratory rate $>30$ breaths/min, patients who were referred from ICU, and patients referred from ward who were on oxygen support were assigned as severe disease.

\section{HRCT of chest}

Non-contrast CT scans were performed using 128-slice CT (Lightspeed, GE Medical Systems) in a craniocaudal direction in a single breath-hold, from the lung apices to lateral costophrenic sulci. A helical scan was obtained in supine position with $120 \mathrm{kVp}, 220-260 \mathrm{~mA}$, rotation time $0.5 \mathrm{~s}$, pitch 0.984 , helical thickness $5 \mathrm{~mm}$, and interslice gap $5 \mathrm{~mm}$, and an additional high-resolution reconstruction was done with a thickness of $1.25 \mathrm{~mm}$ and interslice interval of $1.25 \mathrm{~mm}$. Standard protocols for disinfection and sanitization of the scanning rooms and equipment were followed.

\section{Image analysis}

For assessing the COVID-19 disease load in the lungs, a 40-point scoring system described by Yang et al. [8] was followed. Based on cross-sectional anatomy, 18 segments of both lungs were divided into 20 sub-segmental regions (Table 1).
Opacities in 20 lung regions were subjectively evaluated on HRCT images attributing scores of 0,1 , and 2 if parenchymal opacification involved $0 \%$, less than $50 \%$, and equal or more than $50 \%$ of each segmental region, respectively (Fig. 1).

The CT-SS (hence ranging between 0 and 40) was defined as the sum of scores of all the individual segmental regions. The image review and charting of data were done by two radiologists (A.V. and P.K.S.) each having 17 years of experience. Most entries done by the two observers did not show any difference, but in situations of minor differences, the entry was reviewed by a third senior observer (R.C.S.) with 35 years of experience. All three radiologists evaluated the radiological patterns of involvement and determined a predominant pattern of involvement in consensus as (i) groundglass opacity, (ii) consolidation, (iii) fibrotic pattern, or (iv) mixed pattern. Radiologists were blinded to clinical data, laboratory data, and the vaccination status of patients. All the section CT images were reviewed in a "lung window" (window width of 1000 to 2000 Hounsfield units; window level of -700 to -500 Hounsfield units).

\section{Statistical analysis}

Statistics were performed on SPSS ${ }^{\circledR}$ version 22.0 (IBM Corp 2013) using an independent sample Mann-Whitney $U$ test to compare mean values between the two groups and

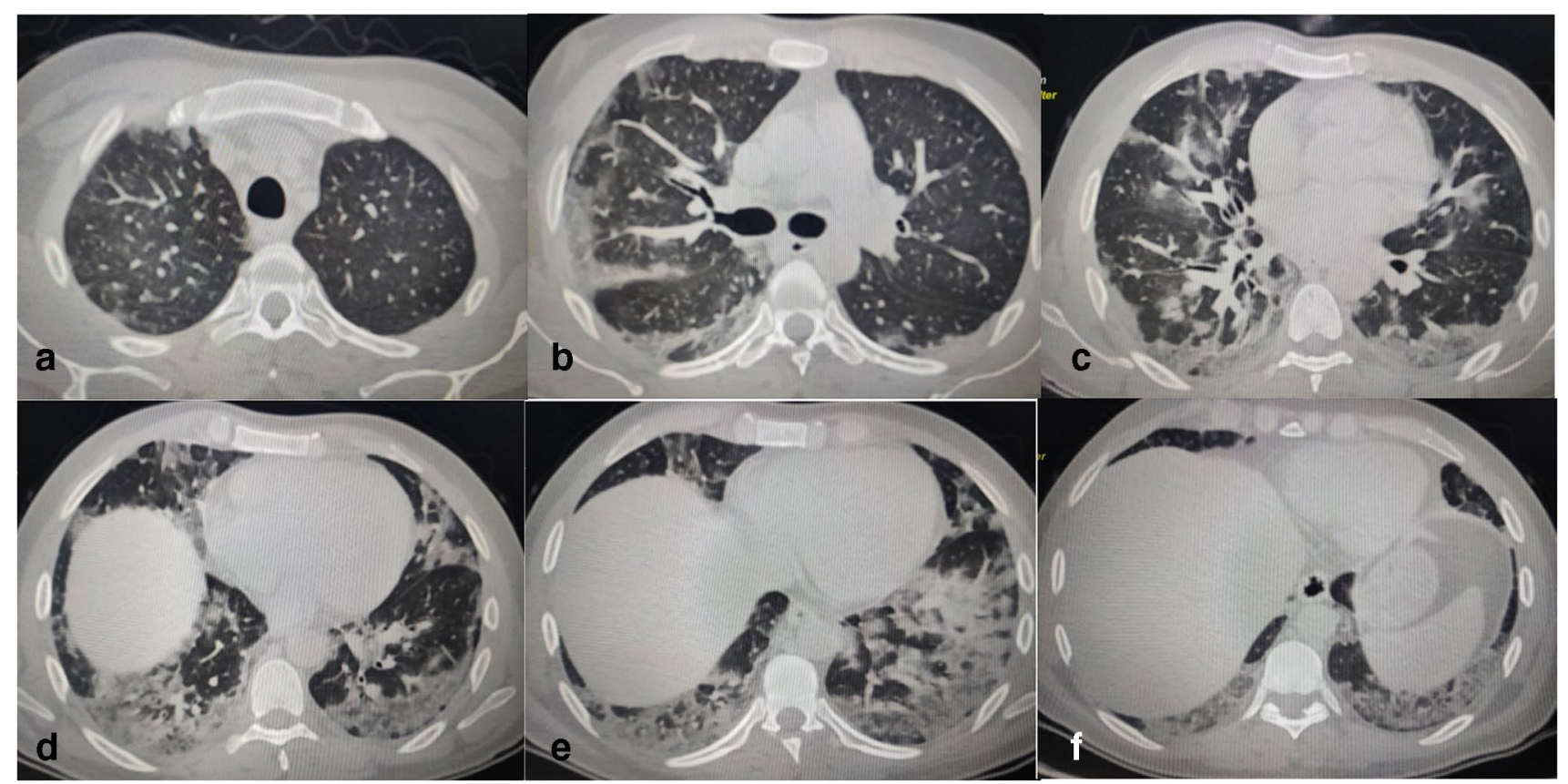

Fig. 1 HRCT thorax (a-f) images in a 36-year-old man with severe COVID-19 pneumonia. a CT images show areas of mixed groundglass opacification and consolidations with segmental scores indicated in the bracket with each segments as follows. a, b Right upper lobe: apical (1), posterior (1), anterior (1) segments and Left upper lobe apical (0), anterior (0), posterior (0). b-f Right middle lobe medial (1) and lateral segments (1) and left upper lobe superior lingular (1) and inferior lingular (2) segments. b-f Right lower lobe superior (1), posterior basal (2), posterior lateral (2), anterior basal (1), and medial basal (1) and left lower lobe: superior (1), posterior basal (2), posterior lateral (2), anterior basal (1), and medial basal (1). A total CT severity score of 22/40 was assigned in this patient 
Kruskal-Wallis test to compare mean between more than two groups. The chi-square test was used for nominal data. A $p$ value of $<0.05$ was considered significant. A logistic regression was performed to ascertain the effects of age, gender, partial vaccination, complete vaccination, and vaccine type on the likelihood that the patients will have severe disease (CT score $\geq 20)$.

\section{Results}

The final dataset consisted of 826 patients (Fig. 2) with a mean age of $44.6 \pm 15.6$ (median 44 years; range 11-87 years) and a male:female ratio of 13:7 $(n=534$ and 292 respectively). The cohort was divided into three groups: (a) the completely vaccinated group receiving two doses of vaccine $(n=49)$, (b) partially vaccinated receiving one dose of vaccine $(n=196)$, and (c) the non-vaccinated group $(n=581)$. Overall, those who had received any vaccination $(n=245)$ had a mean age of $50.0 \pm 14.7$ years (median 52 years; range 21-87 years) and the non-vaccinated group $(n=581)$ had a mean age of $42.4 \pm 15.4$ years (median 40 years; range $11-88$ years). Thirty-six patients had received inactivated viral vaccine (complete vaccination $=7$; incomplete vaccination $=29$ ) while 209 patients received non-replicating viral vector vaccine (complete vaccination $=42$; incomplete vaccination $=167$ ). Of the total 826 patients, 264 patients had clinically severe disease of which 191 were non-vaccinated, 68 were incompletely vaccinated, and 5 were completely vaccinated. Of these 5 patients in the completely vaccinated group, only one needed ICU management and ventilator support. Of these 264 patients, a CT score $\geq 20$ was seen in 165 patients. The sensitivity and specificity of CT severity score equal to or greater than 20 in the prediction of clinically severe disease in our study participants were $62.5 \%$ and $98 \%$, respectively, whereas positive and negative predictive values were $96.4 \%$ and $85.8 \%$ respectively.

The mean CT score of all the patients included in the study was $9.7 \pm 11.1$. The difference between the mean CT score between the vaccinated and non-vaccinated groups was not significant ( $p=0.353$ ) (Table 2$)$. However, the patients who received complete vaccination had significantly low meant CT scores $(3.5 \pm 6.3)$ in comparison to the incompletely vaccinated patients $(10.1 \pm 10.5)$ and non-vaccinated $(10.1 \pm 11.4)$ patients. Patients who had received both

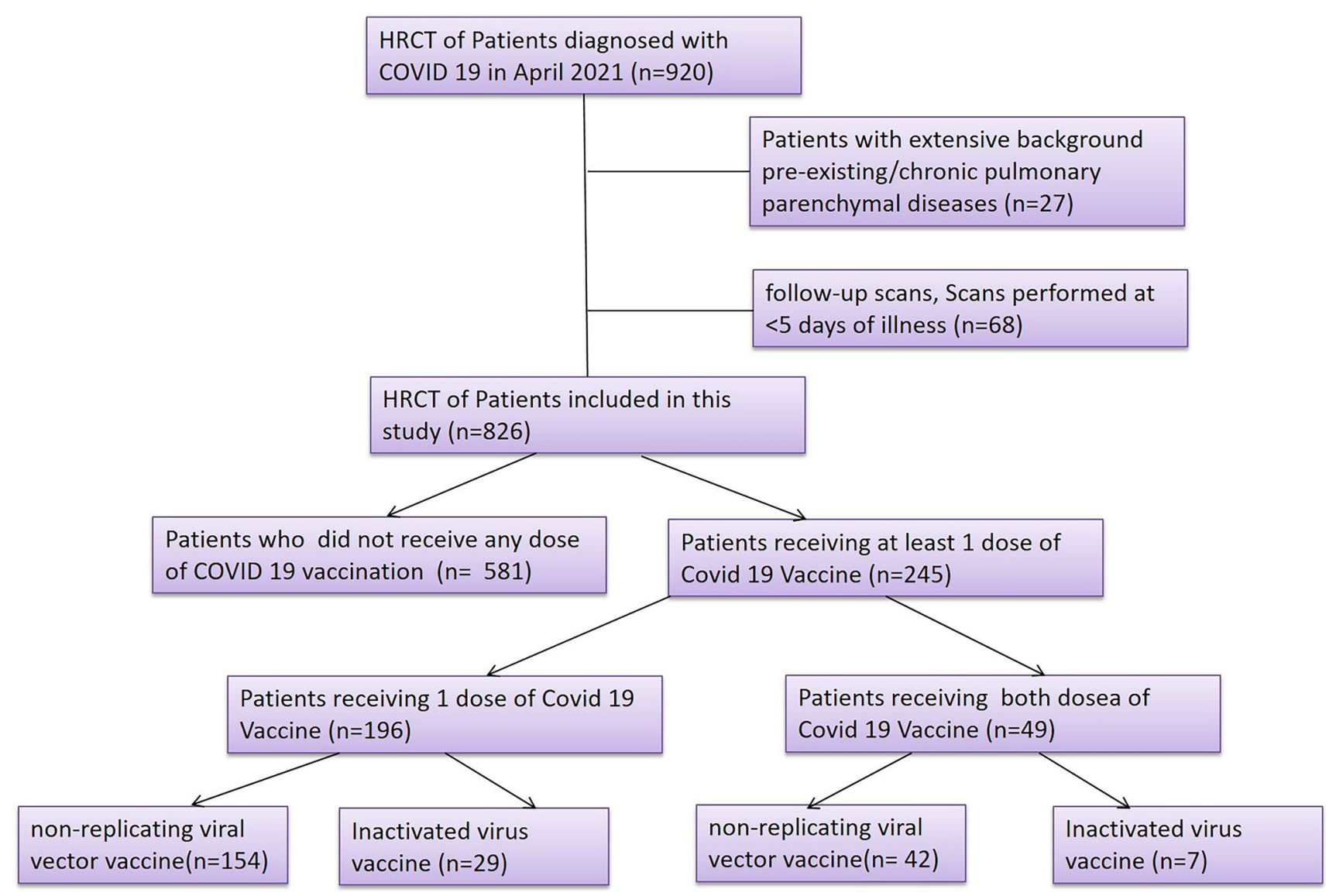

Fig. 2 Flow diagram showing patient enrolment in our study 
Table 2 Multistage intergroup and subgroup analysis among the three groups

\begin{tabular}{|c|c|c|c|}
\hline Groups & No of patients $(n)$ & CT severity score (mean $\pm \mathrm{SD})$ & $p$ value \\
\hline \multicolumn{4}{|l|}{ Non-vaccinated versus vaccinated patients } \\
\hline Non-vaccinated & 581 & $10.1 \pm 11.5$ & \multirow[t]{2}{*}{$0.353^{1}$} \\
\hline Vaccinated & 245 & $8.8 \pm 10.1$ & \\
\hline \multicolumn{4}{|c|}{ Intergroup analysis: non-vaccinated versus partially vaccinated versus completely vaccinated patients } \\
\hline Group A—non-vaccinated & 581 & $10.1 \pm 11.4$ & \multirow{3}{*}{$\begin{array}{r}<\mathbf{0 . 0 0 1}^{2} \\
\quad 0.99(A \text { versus } B) \\
0.001(B \text { versus } C) \\
<0.001(A \text { versus } C)\end{array}$} \\
\hline Group B-incomplete (1 dose) vaccination & 196 & $10.1 \pm 10.5$ & \\
\hline Group $\mathrm{C}$-complete (2 doses) vaccination & 49 & $3.5 \pm 6.3$ & \\
\hline \multicolumn{4}{|l|}{ Subgroup analysis: group C } \\
\hline Within 2 weeks & 14 & $6.8 \pm 9.6$ & \multirow[t]{2}{*}{$0.196^{1}$} \\
\hline More than 2 weeks & 35 & $2.1 \pm 4.4$ & \\
\hline \multicolumn{4}{|c|}{ Subgroup analysis: vaccine sub-types (groups B and C) } \\
\hline Non-replicating viral vector vaccine & 209 & $7.7 \pm 9.4$ & \multirow[t]{2}{*}{$\mathbf{0 . 0 1 2}{ }^{1}$} \\
\hline Inactivated virus vaccine & 36 & $14.5 \pm 12.6$ & \\
\hline \multicolumn{4}{|c|}{ Subgroup analysis: vaccine sub-types (group C only) } \\
\hline Non-replicating viral vector vaccine & 42 & $2.4 \pm 4.3$ & \multirow[t]{2}{*}{$\mathbf{0 . 0 4 7 ^ { 1 }}$} \\
\hline Inactivated virus vaccine & 7 & $7.7 \pm 11.7$ & \\
\hline
\end{tabular}

vaccine doses more than 2 weeks ahead of falling ill $(n=35)$ showed a further lower mean CT severity score (2.1 \pm 4.4$)$ in comparison to those $(n=14)$ who had symptoms within 2 weeks of vaccination $(6.8 \pm 9.6)$. Comparison between the two vaccine types showed that the mean CT severity score was higher in patients receiving inactivated virus vaccine $(14.5 \pm 12.6)$ as compared to those receiving non-replicating viral vector vaccine $(7.7 \pm 9.4)$.

Age-based sub-classification and intergroup comparison (Table 3) of the mean CT scores between the three groups (non-vaccinated, incompletely vaccinated, and completely vaccinated) revealed the mean scores to be significantly lower in completely vaccinated patients of lower ages $(<60$ years) while above that age group the difference was not significant (Table 3; Fig. 3b). The scatter plot (Fig. 3a) between the $\mathrm{CT}$ score and age in the three groups shows a linear relationship between the two variables. For patients, younger than 60 years old, the mean CT-SS was lower $(1.3 \pm 2.4)$ in patients receiving 2 doses more than 2 weeks ago $(n=30)$ compared to those who were vaccinated $(n=8)$ with 2 doses within 2 weeks (mean score $4.6 \pm 4.5$ ). There were no significant differences in mean $\mathrm{CT}$ scores between males and females in both the vaccinated and non-vaccinated groups.

An evaluation of various qualitative radiological findings noted in this cohort among groups with different vaccination statuses was also done. The presence of groundglass opacities and consolidation was significantly lower in patients with complete vaccination compared to nonvaccinated or partially vaccinated patients. The presence of other findings such as fibrosis, nodules, discoid atelectasis, nodules with halo, and pleural effusion was similar between vaccinated and non-vaccinated patients. Enlarged mediastinal lymph nodes (more than $1 \mathrm{~cm}$ in short axis) were found in 7 non-vaccinated patients and were not seen in any patients receiving a vaccination (Table 4$)$. The patients were divided between mild (CT score $<20)$ and severe disease (CT score $\geq 20$ ). We found that only 1 patient who received both doses of vaccine had severe disease, whereas the incidence of severe disease (based on CT score) was significantly higher in the incomplete vaccine $(n=38)$ and non-vaccinated patients $(n=132)$ ( $p$ value $=0.003$; Likelihood ratio $=17.1$ ). There was no significant difference between various qualitative findings between completely vaccinated patients that become symptomatic more than 2 weeks after the 2 nd dose, and those that become symptomatic within 2 weeks. We also evaluated the predominant radiological pattern in the three groups and found that predominant consolidation was most common in the non-vaccinated group (23\%) and least common in the completely vaccinated group (6\%). Predominant ground-glass opacity pattern was most common in the partially vaccinated group, and mixed pattern (both consolidation and ground-glass opacity) was the most common patterns in the non-vaccinated and completely vaccinated groups. 
Table 3 Age and gender based sub-group analysis among the three groups

\begin{tabular}{|c|c|c|c|}
\hline Groups & $\begin{array}{l}\text { No. of } \\
\text { patients }(n)\end{array}$ & $\begin{array}{l}\text { CT severity score } \\
(\text { mean } \pm \text { SD) }\end{array}$ & $p$ value \\
\hline \multicolumn{4}{|c|}{ Age-based sub-group analysis } \\
\hline \multicolumn{4}{|c|}{ Age $<30$} \\
\hline Non-vaccinated & 131 & $4.6 \pm 8.2$ & $0.181^{1}$ \\
\hline Vaccinated & 28 & $1.9 \pm 3.7$ & \\
\hline \multicolumn{4}{|l|}{ Age $30-45$} \\
\hline Non-vaccinated & 204 & $8.5 \pm 10.8$ & $\mathbf{0 . 0 0 3}^{1}$ \\
\hline Vaccinated & 57 & $3.9 \pm 6.8$ & \\
\hline \multicolumn{4}{|l|}{ Age 46-60 } \\
\hline Non-vaccinated & 151 & $14.1 \pm 12.1$ & $\mathbf{0 . 0 3 4 ^ { 1 }}$ \\
\hline Vaccinated & 76 & $9.9 \pm 9.9$ & \\
\hline \multicolumn{4}{|l|}{ Age $>60$} \\
\hline Non-vaccinated & 95 & $14.8 \pm 11.6$ & $0.385^{1}$ \\
\hline Vaccinated & 84 & $13.1 \pm 11.1$ & \\
\hline \multicolumn{4}{|c|}{ Gender-based sub-group analysis } \\
\hline \multicolumn{4}{|l|}{ Male } \\
\hline Non-vaccinated & 373 & $10.7 \pm 11.9$ & $0.154^{1}$ \\
\hline Vaccinated & 161 & $8.5 \pm 9.8$ & \\
\hline \multicolumn{4}{|l|}{ Female } \\
\hline Non-vaccinated & 208 & $9.2 \pm 10.5$ & $0.708^{1}$ \\
\hline Vaccinated & 84 & $9.5 \pm 10.8$ & \\
\hline \multicolumn{4}{|l|}{ Vaccinated group } \\
\hline Male & 161 & $8.5 \pm 9.8$ & $0.450^{1}$ \\
\hline Female & 84 & $9.5 \pm 10.8$ & \\
\hline \multicolumn{4}{|c|}{ Non-vaccinated group } \\
\hline Male & 373 & $10.7 \pm 11.9$ & $0.359^{1}$ \\
\hline Female & 208 & $9.2 \pm 10.5$ & \\
\hline
\end{tabular}

${ }^{1}$ Mann-Whitney $U$ test

$p$ values $<0.05$ are considered significant (bold)

Details of logistic regression analysis are presented in Table 5. Lower age, complete vaccination status, and administration of non-replicating viral vector vaccine were significantly associated with a decreased likelihood of getting a severe disease (CT score $\geq 20$ ). Patients receiving 2 doses of vaccine were 14.29 times less likely to get a severe disease.

\section{Discussion}

In this study, we performed a retrospective analysis of a cohort of vaccinated COVID-19 patients and compared them to non-vaccinated patients scanned in the same time interval to observe the role of the vaccine on CT severity score. This study's major findings were twofold: first, the fully vaccinated patients who did receive two doses of vaccine had a lower mean CT score than unvaccinated and incompletely vaccinated patients; secondly, the completely vaccinated patients who received both doses more than 2 weeks ago from the onset of clinical symptoms of COVID 19 had a lower CT score than those who were vaccinated less than 2 weeks ago.

Various studies have assessed the safety and efficacy of various vaccines that are being administered and [9-19] and have shown that the administration of vaccine scales down the pulmonary involvement in COVID along with an effect on the systemic inflammatory and coagulopathic responses. Our data provides substantial evidence of effectiveness of the vaccines against severe disease with CT severity score considered an imaging surrogate of the biological activity of the virus. The time interval between the second dose and clinical illness of more than 2 weeks was a significant factor resulting in lower CT severity scores in our study, similar to other studies which evaluated the patients of clinic-pathological parameters only [15-19].

Four stages of evolution of COVID have been identified on HRCT: early stage (0-4 days); progressive stage (5-8 days); peak stage (10-13 days); and absorption stage ( $\geq 14$ days) [7]. Imaging was performed for assessment of severity in most of the patients on the 5th-8th day (progressive stage) as per our Institute's protocol. We have included the scans performed in this stage in our study as the inclusion of the scans performed in other stages could have a potential confounding effect on interpretation.

The immune response in females has been considered to be superior to that in males both to internal and external antigens [20]. This physiological advantage reflects as much in recovery from diseases as the inefficacy of vaccines to multiple pathogens [21]. The present dataset however shows a paradoxical but insignificantly higher $\mathrm{CT}$ severity score in vaccinated females compared to the non-vaccinated females whereas in male vaccinated group showed a marginally lower mean CT score. Gender-based intergroup comparisons (Table 3) however did not yield any significant differences. This may be because the immunological response is known to be affected by the overall nutritional status of a person, which is poorer in females in this region as compared to the developed nations where studies advocating the above notion [20-22] have been conducted. Elderly patients are known to have a poorer outcome with multiple factors being held responsible for a more severe affliction of pulmonary parenchyma in them [23]. CT scan correlates of this clinical assertion have also confirmed the notion with milder and more atypical features seen at younger ages [24-27]. In the present dataset, a comparison between mean CT severity score at various age groups between completely vaccinated, incompletely vaccinated, and non-vaccinated patients was in confirmation to earlier studies with a lower mean score noted in patients less than 45 years. An inverse relation between the age and CT score was more prominent in the group which had received complete vaccination. 


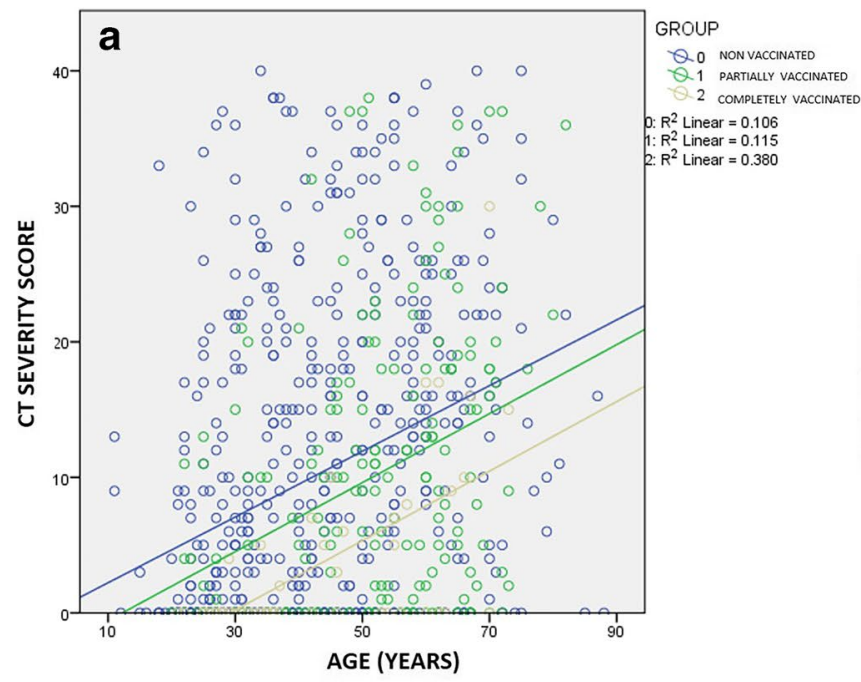

Fig. 3 a Scatter plot showing correlation of age (in years) with the CT severity score. The central tendency in all three groups shows an ascending trend for the CT-SS as the age increases. Though all groups show a second-order linear correlation, a steeper slope with a higher central tendency is seen in the non-vaccinated group. $\mathbf{b}$

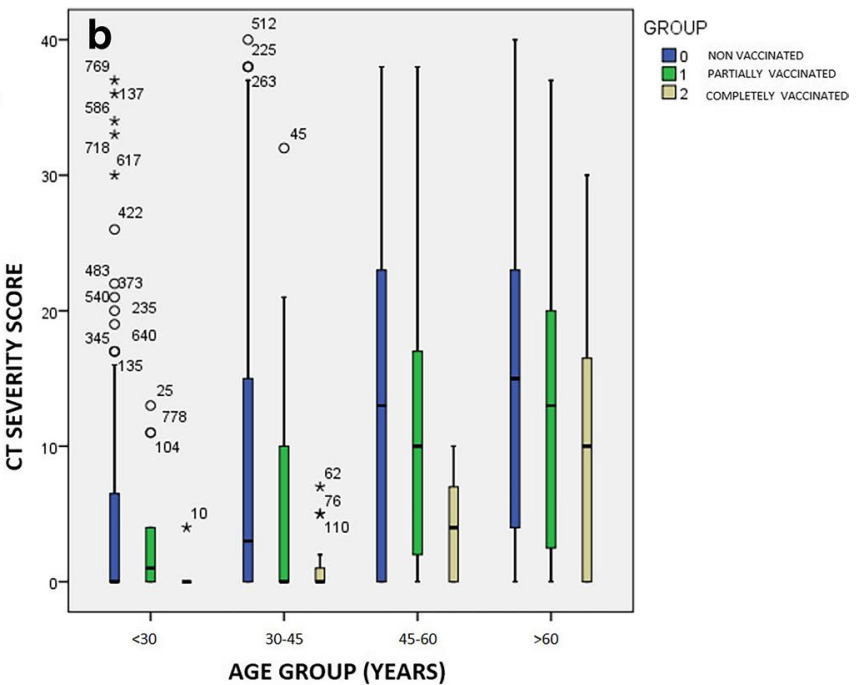

Box-whisker analysis of the CT-SS among the groups shows the nonvaccinated patients to have a higher median value in all sub-groups below 60 years of age. Note that the positive outliers are more in number in the non-vaccinated patients
Table 4 Distribution of qualitative CT scan features in each of the sub-groups with differential analysis of significance

\begin{tabular}{llllc}
\hline Radiological features & $\begin{array}{l}\text { Non-vaccinated } \\
(n=581)\end{array}$ & $\begin{array}{l}\text { Partially vacci- } \\
\text { nated }(n=196)\end{array}$ & $\begin{array}{l}\text { Completely vac- } \\
\text { cinated }(n=49)\end{array}$ & $p$ value \\
\hline Ground-glass opacity & 339 & 130 & 18 & $<\mathbf{0 . 0 0 1}$ \\
Consolidation & 267 & 81 & 6 & $<\mathbf{0 . 0 0 1}$ \\
Fibrosis & 17 & 10 & 1 & 0.49 \\
Nodules & 11 & 3 & 0 & 0.6 \\
Halo around nodules & 2 & 2 & 0 & 0.42 \\
Pleural effusion & 19 & 2 & 1 & 0.24 \\
Discoid atelectasis & 44 & 18 & 2 & 0.44 \\
Mediastinal lymphadenopathy & 7 & 0 & 0 & 0.23 \\
Predominant radiological pattern & of involvement & & & 0.001 \\
$\quad$ Ground-glass opacity & $208(36 \%)$ & $96(49 \%)$ & $13(27 \%)$ & \\
$\quad$ Consolidation & $136(23 \%)$ & $30(15 \%)$ & $3(6 \%)$ & $2(4 \%)$ \\
$\quad$ Fibrosis & $12(2 \%)$ & $7(4 \%)$ & $31(63 \%)$ & \\
$\quad$ Mixed & $225(39 \%)$ & $63(32 \%)$ & \\
\hline
\end{tabular}

Chi-square test

$p$ values $<0.05$ are considered significant (bold)

Table 5 The results of binomial logistic regression to ascertain the effects of age, gender, partial vaccination, complete vaccination, and vaccine type to predict severe disease (CT-SS $\geq 20$ )

\begin{tabular}{lccccc}
\hline Variables & Estimate & SE & Significance & Odds ratio & 95\% confidence interval \\
\hline Gender (male) & .108 & .190 & .569 & .89 & $0.61-1.31$ \\
Age & .035 & .006 & $\mathbf{< . 0 0 1}$ & 1.04 & $1.03-1.05$ \\
Partial vaccination & -1.49 & 1.532 & .334 & 4.45 & $0.21-92.48$ \\
Complete vaccination & -2.66 & 1.151 & $\mathbf{. 0 2 1}$ & 14.29 & $1.49-136.38$ \\
Inactivated virus vaccine & -2.024 & 1.607 & 0.208 & 7.57 & $0.324-176.77$ \\
Non-replicating viral vector vaccine & -1.562 & .449 & $\mathbf{. 0 0 1}$ & 4.77 & $1.98-11.51$ \\
\hline
\end{tabular}

$p$ values $<0.05$ are considered significant (bold) 
Our study also showed that acute air-space and interstitial inflammation characterized by consolidations and ground-glass opacities were significantly lower in the group receiving complete vaccination as compared to the unvaccinated and incompletely vaccinated patients. Accordingly, severe score (CT score $\geq 20$ ) was observed in only a solitary patient who was completely vaccinated and was seen in a greater number of patients who were unvaccinated and incompletely vaccinated. No significant difference in other morphological (sub-acute and chronic) features was however noted among the groups. However, consolidation as a predominant radiological pattern was least common in the completely vaccinated group while it was most common in the non-vaccinated group. This finding may have a potential prognostic value as presence of a consolidation predominant radiological pattern on initial CT chest has been commonly observed in critically ill patients [28].

The present dataset did not chart certain other factors like the presence of co-morbidities, substance abuse, and daily activity levels, which could have possibly affected the severity of affliction of pulmonary parenchyma by SARS$\mathrm{CoV}-2$. Since all the patients included in this study belonged to same region, hence it seems unlikely that these factors would have been significantly different among individuals in the cohort. Further, in the interest of time and an urgent need to document information related to the SARS-CoV-2 vaccine, we did not consider the analysis of serial scans; this may be of interest to the scientific community especially for correlating the vaccination dose, interval, and type of vaccine with the CT severity score and morphological changes. The low number of patients is also a limitation of the study, especially in the group receiving inactivated virus vaccines. Another limitation of this study is that we did not evaluate the inter-observer agreement between the two radiologists in the study. Finally, we did not correlate the CT severity scores with the final outcome because the outcome was affected by many confounding factors such as limited availability of hospital beds and supply of medications during the pandemic.

\section{Conclusion}

In this retrospective study, patients receiving both doses of the SARS-CoV-2 vaccine showed lower CT severity scores in comparison to those who received only one dose or those who did not receive the vaccine at all. The time elapsed between vaccination and onset of clinical symptoms affects the pulmonary changes as seen on HRCT of the lung, with the patients who developed COVID symptoms 2 weeks or more after the second dose of vaccine showing lower CT severity scores. With CT severity score as the bio-surrogate, both vaccines analyzed together performed better in completely vaccinated patients less than 45 years of age in comparison to older patients. Finally, the pulmonary HRCT changes were more severe in non-vaccinated and incompletely vaccinated patients as compared to the completely vaccinated individuals.

Funding The authors state that this work has not received any funding.

\section{Declarations}

Guarantor The scientific guarantor of this publication is Prof. Ashish Verma.

Conflict of interest The authors of this manuscript declare no relationships with any companies whose products or services may be related to the subject matter of the article.

Statistics and biometry One of the authors, Dr. Ishan Kumar, has significant statistical expertise.

Informed consent Written informed consent was obtained from all subjects (patients) in this study.

Ethical approval Institutional Review Board approval was obtained.

Methodology
- retrospective
- observational
- performed at one institution

\section{References}

1. Pontone G, Scafuri S, Mancini ME et al (2021) Role of computed tomography in COVID-19. J Cardiovasc Comput Tomogr 15:27-36

2. Bollineni VR, Nieboer KH, Döring S, Buls N, de Mey J (2021) The role of CT imaging for management of COVID-19 in epidemic area: early experience from a University Hospital. Insights Imaging 12:10

3. Hope MD, Raptis CA, Shah A, Hammar MH, Henry TS (2021) A role for CT in COVID-19? What data really tell us so far. Lancet 395(10231):P1189-1190

4. Elicker BM (2021) What is the performance and role of CT in suspected COVID-19 infection? Radiology 298(2):E109-E111

5. Ai T, Yang Z, Hou H et al (2020) Correlation of chest CT and RTPCR testing in coronavirus disease 2019 (COVID-19) in China: a report of 1014 cases. Radiology 296:E32-E40

6. American College of Radiology (2020) ACR recommendations for the use of chest radiography and computed tomography (CT) for suspected COVID-19 infection. March 11, 2020. https://www. acr.org/Advocacy-and-Economics/ACR-PositionStatements/ Recommendations-for-Chest-Radiography-and-CT-for-Suspe cted-COVID19-Infection. Accessed 23 Mar 2020

7. Pan F, Ye T, Sun P et al (2020) Time course of lung changes at chest CT during recovery from coronavirus disease 2019 (COVID-19). Radiology 295(3):715-721

8. Yang R, Li X, Liu H et al (2020) Chest CT severity score: an imaging tool for assessing severe COVID-19. Radiol Cardiothorac Imaging 2(2):e200047

9. Haas EJ, Angulo FJ, McLaughlin JM et al (2021) Impact and effectiveness of mRNA BNT162b2 vaccine against SARS-CoV-2 
infections and COVID-19 cases, hospitalisations, and deaths following a nationwide vaccination campaign in Israel: an observational study using national surveillance data. Lancet 397:1819-1829

10. Polack FP, Thomas SJ, Kitchin N et al (2020) Safety and efficacy of the BNT162b2 mRNA covid-19 vaccine. N Engl J Med 383:2603-2615

11. Baden LR, El Sahly HM, Essink B et al (2021) Efficacy and safety of the mRNA-1273 SARS-CoV-2 Vaccine. N Engl J Med 384:403-416

12. Dagan N, Barda N, Kepten E et al (2021) BNT162b2 mRNA Covid-19 vaccine in a nationwide mass vaccination setting. $\mathrm{N}$ Engl J Med 384:1412-1423

13. Anderson EJ, Rouphael NG, Widge AT et al (2020) for mRNA1273 Study Group. Safety and immunogenicity of SARSCoV-2 mRNA-1273 vaccine in older adults. N Engl J Med 383(25):2427-2438

14. Walsh EE, Frenck RW Jr, Falsey AR et al (2020) Safety and immunogenicity of two RNA-based Covid-19 vaccine candidates. N Engl J Med 383(25):2439-2450

15. Hill EM, Keeling MJ (2021) Comparison between one and two dose SARS-CoV-2 vaccine prioritization for a fixed number of vaccine doses. Medrxiv 03(15):21253542. https://doi.org/10. $1101 / 2021.03 .15 .21253542$

16. Monin L, Laing AG, Muñoz-Ruiz M et al (2021) Safety and immunogenicity of one versus two doses of the COVID-19 vaccine BNT162b2 for patients with cancer: interim analysis of a prospective observational study. Lancet Oncol 22:765-778

17. Callaway E, Mallapaty S (2021) What scientists do and don't know about the Oxford-Astrazeneca covid vaccine- results confirming that the vaccine provides strong protection against COVID-19 were welcomed after a pause in roll-outs. Nature 592:15-17

18. Callaway E (2020) Why Oxford's positive COVID vaccine results are puzzling scientist's: preliminary data suggest that the immunization was more effective in trial participants who received a lower dose. Nature 588:16-18
19. Voysey M, Clemens SAC, Madhi SA et al (2021) Single-dose administration and the influence of the timing of the booster dose on immunogenicity and efficacy of ChAdOx $1 \mathrm{nCoV}-19$ (AZD1222) vaccine: a pooled analysis of four randomized trials. Lancet 397:881-891

20. Klein SL, Flanagan KL (2016) Sex differences in immune responses. Nat Rev Immunol 16:626-638

21. McCartney PR (2020) Sex-based vaccine response in the context of COVID-19. J Obstet Gynecol Neonatal Nurs 49:405-408

22. Fathi A, Addo MM, Dahlke C (2020) Sex differences in immunity: implications for the development of novel vaccines against emerging pathogens. Front Immunol 11:601170

23. Mueller AL, McNamara MS, Sinclair DA (2020) Why does COVID-19 disproportionately affect older people? Aging 12(10):9959-9981

24. Ramasamy MN, Minassian AM, Ewer KJ et al (2020) Safety and immunogenicity of ChAdOx $1 \mathrm{nCoV}-19$ vaccine administered in a prime-boost regimen in young and old adults (COV002): a single-blind, randomized, controlled, phase $2 / 3$ trial. Lancet 396:1979-1993

25. Soiza RL, Scicluna C, Thomson EC (2021) Efficacy and safety of COVID-19 vaccines in older people. Age Ageing 50:279-283

26. Azadbakht J, Haghi-Aminjan H, Farhood B (2020) Chest CT findings of COVID-19-infected patients, are there differences between pediatric and adult patients? A systematic review. Egypt J Radiol Nucl Med 51:145

27. Ai T, Yang Z, Hou H et al (2020) Correlation of Chest CT and RTPCR testing for coronavirus disease 2019 (COVID-19) in China: a report of 1014 cases. Radiology 296:E32-E40

28. Tabatabaei SMH, Talari H, Moghaddas F, Rajebi H (2020) CT features and short-term prognosis of COVID-19 pneumonia: a single-center study from Kashan. Radiol Cardiothorac Imaging 2(2):e200130

Publisher's Note Springer Nature remains neutral with regard to jurisdictional claims in published maps and institutional affiliations. 